\title{
Distribuição diamétrica e determinação da altura em plantio de Araucaria angustifolia (Bertol.) Kuntze na região central do Rio Grande do Sul
}

Diametric distribution and height estimation of an Araucaria angustifolia (Bertol.) Kuntze plantation in central region of Rio Grande do Sul

\author{
Â. M. K. Hentz ${ }^{1 *}$; D. L. Pasa $^{2} ;$ M. Talgatti ${ }^{2}$; A. da R. C. Ferreira ${ }^{2}$; J. A. de Mello \\ Filho $^{2}$ \\ ${ }^{1}$ Departamento de Ciências Florestais/Setor de Ciências Agrárias, Universidade Federal do Paraná, CEP 80210- \\ 170 Curitiba-PR, Brasil \\ ${ }^{2}$ Departamento de Engenharia Rural/Centro de Ciências Rurais, Universidade Federal de Santa Maria, CEP $97105-$ \\ 900 Santa Maria-RS, Brasil \\ *angelakhentz@gmail.com
}

(Recebido em 25 de agosto de 2015; aceito em 18 de dezembro de 2015)

\begin{abstract}
O presente trabalho visou determinar matematicamente a curva hipsométrica, assim como a distribuição diamétrica da espécie Araucaria angustifolia, em um plantio localizado na área da FEPAGRO Florestas, Santa Maria - RS. A distribuição diamétrica do povoamento foi modelada a partir de funções de densidade de probabilidade (fdp), e a relação hipsométrica a partir de modelos matemáticos. Após os ajustes, verificou-se que os modelos hipsométricos obtiveram valores de coeficiente de determinação múltipla que variaram de 0,36 até 0,47 , e erro padrão entre 10,58 e 11,64\%. Pela análise realizada, pôdese verificar que o modelo $h=\beta_{0}+b_{1}$ dap $+\beta_{2} d_{a p}{ }^{2}$ foi o mais apto às condições locais, obtendo $R^{2} a j=0,47$ e $\mathrm{Sxy} \%=10,59 \%$. A função SB de Johnson foi a que melhor se ajustou à distribuição diamétrica, conforme o teste de Kolmogorov-Smirnov. Pode-se concluir que as equações hipsométricas podem ser utilizadas para estimar a variável altura da espécie nesta região com erros aceitáveis, assim como pode ser modelada a distribuição diamétrica da floresta a partir de várias funções.
\end{abstract}

Palavras-chave: Análise de regressão, Modelos estimativos, Pinheiro do Paraná.

This study aim to determine the mathematical model that defines the best hypsometric curve of the relationship between height/diameter from the specie of Araucaria angustifolia, in a plantation located in the area of FEPAGRO FORESTS, Santa Maria-RS. The diametric distribution of this plantation was modulate from probability density function (pdf) and relationship height/diameter using mathematic functions. After adjustment, the results showed that the multiple coefficient of determination presents values ranging from 0.36 until 0.47 , and standard error between $10.58 \%$ and $11.64 \%$. When these parameters were analyzed, they shown that the $h=\beta_{0}+b_{1}$ dap $+\beta_{2}$ dap ${ }^{2}$ was the model that had better adapt for the local conditions, obtaining $\mathrm{R}^{2} \mathrm{aj}=0.47$ e Sxy\% $=10.59$. The function SB of Johnson was the one that had better adapt to diametric distribution, according to the Kolmogorov-Smirnov test. The conclusion is that the hypsometric equations can be utilize to estimate the height variable of the specie in the region with acceptable errors, as well as can be modulate the diametric distribution of the forest, utilizing many functions.

Keywords: Regression analysis, estimation models, Paraná pine.

\section{INTRODUÇÃO}

Araucaria angustifolia (Bert.) O. Ktze, mais conhecida como Pinheiro-do-Paraná, é uma espécie considerada de grande valor econômico, paisagístico e cultural. De acordo com Scheeren et al. (1999) [1], a araucária constituiu a mais importante essência florestal nativa da economia madeireira do sul do Brasil e também a mais valiosa conífera de ocorrência natural no País. No entanto, conforme dados da Secretaria de Estado de Meio Ambiente e Recursos Hídricos (SEMA) e do Instituto Ambiental do Paraná (IAP), apesar da importância da espécie, a mesma encontra-se na lista de espécies ameaçadas de extinção. A exploração excessiva, sem a devida reposição, tem sido apontada como a principal razão dessa ameaça [2]. Contudo, a falta 
de conhecimento do comportamento e dos requisitos silviculturais da espécie são fatores que podem favorecer sua extinção [1].

Para minimizar os danos e desenvolver uma alternativa conservacionista e economicamente viável, estão sendo realizados estudos e experimentos com plantios de araucária a fim de aprimorar e conhecer seu comportamento em relação, principalmente, ao seu crescimento e produção.

Dessa forma, a distribuição diamétrica, caracterizada como sendo a distribuição do número de árvores por hectare (N/ha) por classe de diâmetro (DAP, diâmetro a altura do peito), é de fundamental importância para analisar uma comunidade florestal. Nesse sentido, a distribuição diamétrica permite analisar o estado em que se encontra a floresta, assim como fazer inferências sobre a descontinuidade das classes diamétricas e as características ecofisiológicas das espécies [3]. Além do que, no sentido que permite o conhecimento da estrutura e das taxas de crescimento das espécies, a distribuição diamétrica pode ser utilizada como base para o manejo florestal [4].

Normalmente os povoamentos equiâneos apresentam a curva de distribuição diamétrica com tendência a unimodalidade, com as maiores frequências nos valores médios e as menores nos valores extremos [5], diferentemente do encontrado em florestas tropicais multiâneas, em que se observa a distribuição do tipo "J" invertido, de forma que as maiores frequências encontram-se nas menores classes de DAP [4].

Da mesma forma, as curvas hipsométricas são de grande auxílio para avaliar o processo de desenvolvimento de povoamentos de araucárias, destinados tanto para fins ecológicos quanto econômicos.

Além disso, a medição da altura é um dos processos mais caros de um inventário [6] devido principalmente ao tempo necessário para sua medição. Assim, Azevedo et al. (2011) [7] comentam que, como medir a altura das árvores é um processo exaustivo e sujeito a erros, é pertinente empregar métodos estimativos ou equações hipsométricas. Essas relações foram propostas por Ker e Smith, para minimizar os custos da medição de todas as alturas do povoamento.

Povoamentos plantados, bem conduzidos e manejados, e que se encontram sobre sítios bem definidos, apresentam curva hipsométrica tendencialmente íngreme, ou seja, existe uma alta correlação entre as variáveis altura e diâmetro. Entretanto, em populações naturais, que se localizam em condições de sítios diversos, não conduzidos e não manejados, têm a propriedade de apresentar pontos dispersos na curva hipsométrica, ou seja, de mostrar uma baixa correlação entre diâmetro e altura, evidenciando assim maior heterogeneidade na população [8]. Apesar disso, o uso de equações hipsométricas no inventário tem sido cada vez mais frequente, tornando-o mais econômico e, na maioria das vezes, tão preciso quanto o realizado medindo-se a altura de todas as árvores da parcela [9].

Em vista da importância de um bom planejamento do plantio da araucária, e consequentemente o bom desenvolvimento do mesmo, torna-se de suma importância estudar as relações biométricas da população. Sendo assim, este estudo visou determinar o modelo matemático que melhor delimite a curva hipsométrica assim como a distribuição diamétrica da espécie Araucaria angustifolia (Bertol.) como subsídio ao manejo econômico, sustentável e produtivo dos cultivos florestais da mesma na região central do Estado do Rio Grande do Sul.

\section{MATERIAL E MÉTODOS}

\subsection{Caracterização e descrição da área de estudo}

O estudo foi realizado em uma área plantada com Araucaria angustifolia (Bertol.) Kuntze no

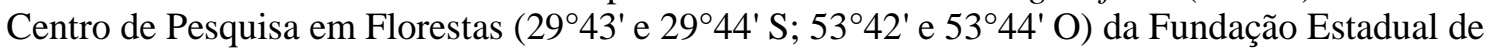
Pesquisa Agropecuária do Rio Grande do Sul (FEPAGRO-RS). Este centro está localizado no município de Santa Maria-RS, no distrito de Boca do Monte a cerca de $16 \mathrm{~km}$ a noroeste do centro da zona urbana e encontra-se aproximadamente a $100 \mathrm{~m}$ de altitude.

A região de Santa Maria está situada praticamente na zona de transição entre a Depressão Periférica Sul-Rio-Grandense, também conhecida por Depressão Central, e a escarpa arenitobasáltica do Planalto Meridional Brasileiro [10]. 


\subsection{Base de dados}

Neste estudo foram utilizados dados de 673 indivíduos de Araucaria angustifolia obtidos por meio de um censo realizado em um povoamento com idade aproximada de 60 anos. Neste povoamento não foi realizado nenhum tipo de desrama ou de desbaste sendo que, através do levantamento a campo, observou-se um grande número de árvores suprimidas e, consequentemente, com alturas relativamente baixas.

Foram mensuradas as variáveis CAP (circunferência a $1,30 \mathrm{~m}$ de altura do solo, conhecida como altura do peito) e altura total. Como muitos indivíduos mensurados estavam mortos ou em processo avançado de deterioração, estes foram excluídos das análises, trabalhando-se com dados de 508 indivíduos.

\subsection{Distribuição diamétrica}

Para modelar a distribuição diamétrica do povoamento de araucárias, os indivíduos foram distribuídos em dez classes de DAP com amplitude de $6,55 \mathrm{~cm}$ cada. O número de classes foi derivado da fórmula de Sturges.

A distribuição diamétrica da floresta foi ajustada a fim de seis funções de densidade de probabilidade (fdp), para se estimar a frequência de indivíduos em cada classe de DAP considerada. As funções utilizadas foram: Normal, Log-normal, Weber, Weibull 3 parâmetros, Gama e SB de Johnson. O ajuste foi obtido pela regressão não linear utilizando-se o software Table Curve versão 3. As fórmulas matemáticas das funções podem ser visualizadas na Tabela 1. Depois de ajustadas, as funções foram avaliadas pelo teste de aderência de KolmogorovSmirnov (KS) ao nível de 95\% de probabilidade.

Tabela 1: Funções Probabilísticas ajustadas e testadas para modelar a distribuição diamétrica de Araucaria angustifolia

\begin{tabular}{|c|c|c|}
\hline Função & Expressão Matemática & Condicionantes \\
\hline Normal & $f(x)=\frac{1}{\sigma \sqrt{2 \pi}} e^{-\frac{(x-\mu)^{2}}{2 \sigma^{2}}}$ & $\begin{aligned} \sigma & >0 \\
-\infty & <x<+\infty \\
-\infty & <\mu<+\infty\end{aligned}$ \\
\hline Weber & $f(x)=\frac{x^{a}}{(b+c x)^{d}}$ & $\begin{array}{c}0 \leq x<+\infty \\
d \geq a+1 \\
d \neq a+2 \\
d \neq a+3 \\
a, b, c, d>0\end{array}$ \\
\hline Weibull 3P & $f(x)=\frac{\gamma}{\beta}\left(\frac{x-\alpha}{\beta}\right)^{\gamma-1} \exp \left[-\left(\frac{x-\alpha}{\beta}\right)\right]^{\gamma}$ & $\begin{array}{c}x \geq x_{\min } \\
-\infty<x_{\min } \\
<+\infty \\
b, c>0 \\
a \geq 0\end{array}$ \\
\hline Log-normal & $f(x)=\frac{1}{x \sigma \sqrt{2 \pi}} \exp \left[-\frac{(\log x-\mu)^{2}}{2 \sigma^{2}}\right]$ & $\begin{aligned} x & \geq x_{\min } \\
\sigma & >0 \\
-\infty & <x<+\infty \\
-\infty & <\mu<\infty\end{aligned}$ \\
\hline $\mathrm{S}_{\mathrm{B}}$ de Johnson & $f(x)=\frac{\delta}{2 \pi} * \frac{\lambda}{x-\varepsilon \lambda+\varepsilon-x} * \exp \left(-\frac{1}{2} \gamma+\delta * \ln \frac{x-\varepsilon}{\lambda+\varepsilon-x}\right)^{2}$ & $\begin{array}{c}\varepsilon<x<+\lambda \\
-\infty<\gamma<+\infty \\
\gamma>0 \\
\delta>0\end{array}$ \\
\hline Gama & $f(x)=\frac{1}{\beta^{\alpha} \Gamma(\alpha)} x^{\alpha-1} e^{-\frac{x}{\beta}}$ & $\begin{array}{c}x \geq x_{\min } \\
\propto, \beta>0 \\
-\infty<x_{\min } \\
\quad<+\infty\end{array}$ \\
\hline
\end{tabular}


Em que: $f x=$ função de densidade probabilística da variável $x ; x$ variável aleatória; $\mu$ : média dos valores de $x ; \sigma=$ desvio padrão de $x ; x_{\min }=$ valor mínimo de $x ; x_{\operatorname{máx}}=$ valor máximo de $x ; \pi: 3,14159 \ldots ; e$ $=$ constante de Euler; $a, b, c, d, \alpha, \beta, \gamma, \delta, \varepsilon, \lambda=$ parâmetros a serem estimados.

\subsection{Equações hipsométricas ajustadas}

Foram testados dez modelos de relações hipsométricas comumente utilizadas, conforme a Tabela 2, aplicando-se o Método dos Mínimos Quadrados.

Tabela 2: Modelos ajustados para modelar a relação hipsométrica da Araucaria angustifolia

\begin{tabular}{ccc}
\hline $\mathbf{N}^{\mathbf{0}}$ & Modelo & Nome ou autor \\
\hline 1 & $\mathrm{~h}=\beta_{0}+\beta_{1}$ dap & Linear Simples \\
2 & $\mathrm{~h}=\beta_{0}+\beta_{1} \mathrm{dap}^{2}$ & Azevedo \\
3 & $\mathrm{~h}=\beta 0+\beta_{1}$ dap $+\beta_{2} \mathrm{dap}^{2}+\beta_{3} \mathrm{dap}^{3}$ & \\
4 & $\mathrm{~h}=\beta_{0}+\beta_{1} \ln (\mathrm{dap})$ & Henricksen \\
5 & $\mathrm{~h}=\beta_{0}+\mathrm{b}_{1}$ dap $+\beta_{2} \mathrm{dap}^{2}$ & Trorey \\
6 & $\ln \mathrm{h}=\beta_{0}+\beta_{1} \ln (\mathrm{dap})$ & Stoffels \\
7 & $\ln \mathrm{h}=\beta_{0}+\beta_{1} \mathrm{dap}^{2}$ & \\
8 & $\operatorname{lnh}=\beta_{0}+\beta_{1} \ln$ dap $+\beta_{2} \mathrm{dap}^{-1}$ & Silva (1980) (apud [11]) \\
9 & $\ln \mathrm{h}=\beta_{0}+\beta_{1} * \ln \mathrm{dap}^{2}$ & \\
10 & $\mathrm{~h}=\beta_{0}+\beta_{1} \mathrm{dap}^{-1}$ & Assmann \\
\hline
\end{tabular}

Em que: $\mathrm{h}=$ altura total $(\mathrm{m}) ; \beta_{0}, \beta_{1}, \beta_{2}$ e $\beta_{3}=$ coeficientes da regressão; dap = diâmetro $1,30 \mathrm{~m}$ de altura do solo; $\ln =$ logaritmo neperiano.

As equações foram testadas pelos parâmetros: Coeficiente de Determinação Múltipla Ajustado ( $\mathrm{R}^{2}$ aj), Índice de Ajuste de Schlaegel (IA), Erro Padrão da Estimativa ( $\mathrm{S}_{\mathrm{yx}}$ e $\mathrm{S}_{\mathrm{yx}} \%$ ), e Análise Gráfica de Resíduos.

Foi utilizado o Fator de Correção de Meyer para a correção da discrepância logarítmica nos modelos em que a variável dependente sofreu transformação logarítmica. Da mesma forma, os modelos com transformação logarítmica tiveram seus erros recalculados para a escala original.

\section{RESULTADOS E DISCUSSÃO}

\subsection{Distribuição diamétrica}

Os valores de altura variaram entre 12,8 e $26,1 \mathrm{~m}$ com média em 19,67 m e desvio padrão de 2,86 m. Já os diâmetros apresentaram maior variação, entre 7,63 e 73,21 cm, média de 33,27 $\mathrm{cm}$, e desvio padrão de $9,94 \mathrm{~cm}$. Este fato explica-se por ser a variável diâmetro fortemente afetada por parâmetros como a densidade da floresta [12].

A distribuição diamétrica dos dados originais apresentou valor de curtose de 0,3728 e de assimetria 0,4055 , indicando que a curva tem assimetria positiva, e apresenta achatamento (curtose superior a 0,263). Segundo Machado et al. (2006) [13], com o envelhecimento do povoamento a curva de distribuição diamétrica tende a apresentar assimetria à direita (ou negativa) e maior achatamento. Dessa forma, verifica-se que o povoamento ainda não está estagnado, pois existe maior número de indivíduos nas menores classes de DAP e o achatamento não é muito elevado.

Foi possível ajustar todas as seis fdp testadas, e os parâmetros das estatísticas dos ajustes estão apresentados na Tabela 3, juntamente com os valores do teste de Kolmogorov-Smirnov. 
Tabela 3: Ajustes das funções de densidade de probabilidade para a distribuição diamétrica de Araucaria angustifolia

\begin{tabular}{cccccc}
\hline Funções & $\begin{array}{c}\text { Frequência total } \\
\text { estimada }\end{array}$ & D calc. & D tab. 95\% & $\mathrm{R}_{\text {aj. }}^{2}$ & Ranking \\
\hline Normal & 505 & 0,02440 & 0,06034 & 0,98 & 5 \\
Weber & 503 & 0,01984 & 0,06034 & 0,99 & 3 \\
Weibull 3P & 507 & 0,02165 & 0,06034 & 0,98 & 4 \\
SB de Johnson & 503 & 0,01329 & 0,06034 & 0,99 & 1 \\
Log-normal & 505 & 0,03105 & 0,06034 & 0,99 & 6 \\
Gama & 505 & 0,01698 & 0,06034 & 0,99 & 2 \\
\hline
\end{tabular}

Em que: D calc. é o valor do teste de Kolmogorov-Smirnov calculado, e D tab. é o valor do do teste de Kolmogorov-Smirnov tabelado.

Observa-se na tabela que todas as funções obtiveram um bom ajuste, pois os valores do teste Kolmogorov-Smirnov calculado, em todas elas, são bem inferiores ao valor tabelado. Dessa forma, qualquer uma dessas funções pode ser utilizada para modelar o comportamento diamétrico deste povoamento.

Como todas as funções aderiram aos dados, foi realizado um ranqueamento destas, pelo qual a melhor função ( $1^{\mathrm{a}}$ colocação) foi a que obteve o menor valor de $\mathrm{D}$ calculado, e a pior $\left(6^{\mathrm{a}}\right.$ colocação), a que obteve o maior valor. Pelo ranking escolheu-se a função SB de Johnson como a fdp que melhor estimou os valores de frequência por classe de DAP deste povoamento. Os valores estimados para essa função, assim como para as outras 5, plotados sobre os dados reais, podem ser visualizados na Figura 1.

A função SB de Johnson é utilizada com muito êxito na área florestal, e foi também considerada a melhor função para modelar a distribuição diamétrica de araucária (quando utilizado intervalo de classe de $5 \mathrm{~cm}$ ) por Machado et al. (2009) [14], demonstrando sua adaptação ao formato de curva unimodal. Da mesma forma, a função SB de Johnson mostrou-se superior à outras distribuições padrão, para modelar a distribuição diamétrica de bracatingais [15], para Ocotea nutans [16], e para Pinus taeda nas idades de 6 a 8 anos [17].

Apesar disso, como os valores entre as funções são muito semelhantes, deve-se considerar também a superioridade da função Weibull-3P na modelagem da distribuição diamétrica deste povoamento, visto que esta função apresentou valor de frequência total estimada mais próxima da frequência real do povoamento, respectivamente de 507 e 508. A Função Weibull-3P foi também considerada a mais adequada por Curto et al. (2014) [18] para modelar a distribuição diamétrica dessa mesma espécie, e é frequentemente utilizada para modelar distribuições diamétricas tanto de povoamentos como de florestas naturais, devido às suas características de flexibilidade de parâmetros, que permitem que a mesma se ajuste desde distribuições decrescentes até distribuições unimodais [19].

A função Weibull-3P, conforme já destacado, apresenta um ótimo ajuste às funções de diversas espécies, podendo-se citar os trabalhos como de Orellana et al. (2014) [20], que analisando os ajustes de 20 espécies encontradas na Floresta Nacional (FLONA) de Irati no Centro-Sul do Paraná, destacaram que a distribuição Weibull-3P foi a mais adequada, levando em consideração o índice de Reynolds e também o Erro-Padrão da Estimativa. Da mesma forma, Amaral et al. (2015) [21] analisaram as funções Beta, Gama e Weibull-3P, ajustadas pelo método da máxima verossimilhança, para as espécies de Eugenia involucrata, Myrciaria cauliflora e Ilex paraguariensis oriundas de um fragmento de Floresta Ombrófila Mista no estado do Paraná e, segundo os autores, foi possível destacar a função densidade de probabilidade Weibull-3P como sendo a que melhor se ajustou aos dados das distribuições diamétricas das espécies estudadas.

O ajuste de uma função que possa modelar a distribuição diamétrica de uma floresta é muito importante, principalmente como ferramenta para projeções futuras da floresta, sendo utilizado com muito sucesso em modelos de predição de crescimento, de recrutamento e até de mortalidade. Segundo Curto et al. (2014) [18], essas funções podem ser utilizadas para projeções futuras desde que o povoamento não sofra intervenções silviculturais. A função SB 
em particular apresenta-se como uma ferramenta muito útil pra a predição da produção futura de plantios homogêneos, como observado para Eucalyptus camaldulensis [22].

As curvas das distribuições diamétricas ajustadas podem ser observadas na Figura 1, em que é possível observar a semelhança das curvas estimativas em relação aos valores observados. Pode-se perceber, ainda, que ocorreu maior concentração de indivíduos nas classes centrais da distribuição diamétrica, o que, segundo Lima \& Leão (2013) [3], é um fator característico de uma curva normal, sendo muito comum em florestas plantadas.
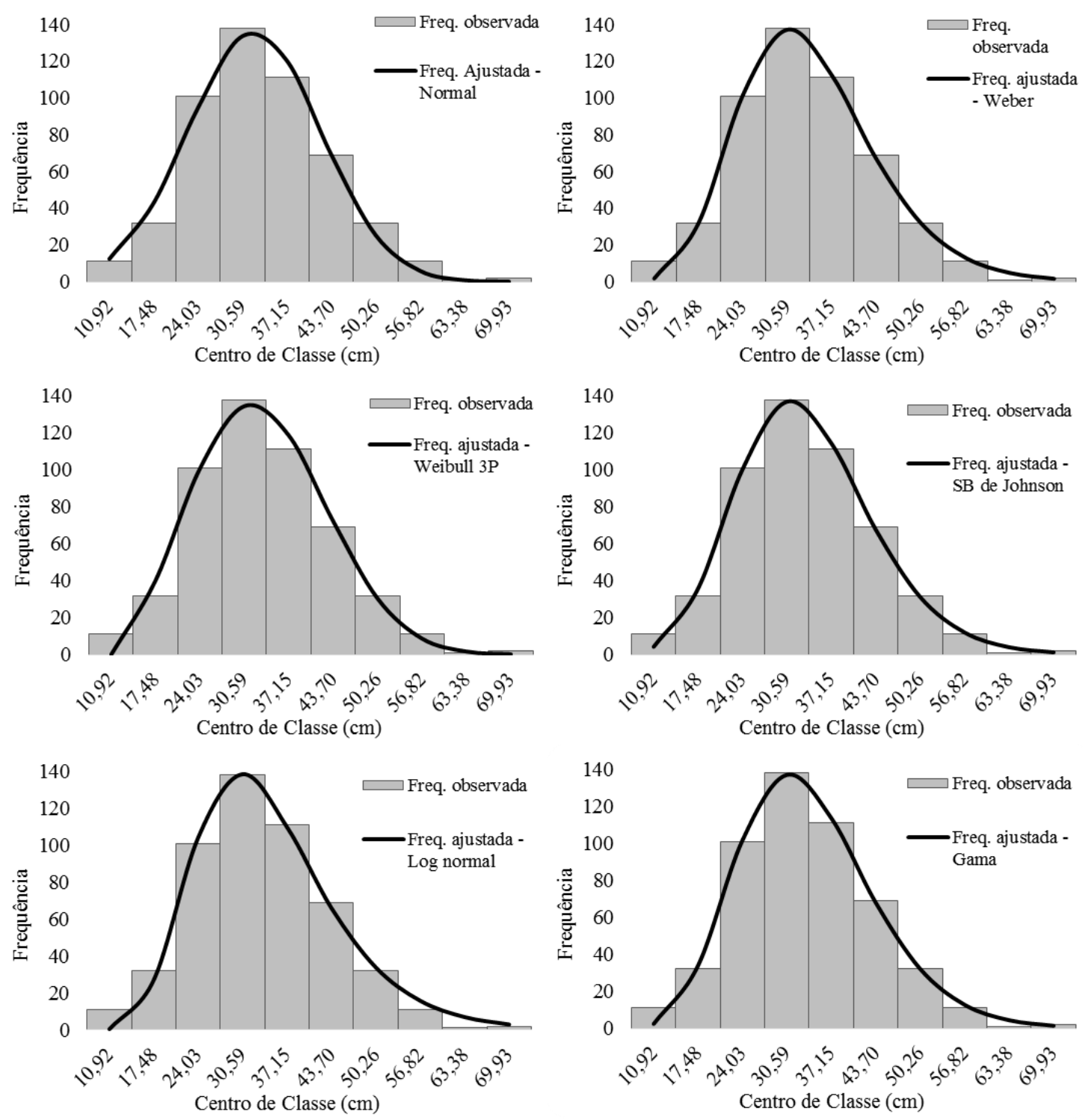

Figura 1: Ajustes das distribuições em comparação com os valores reais de frequências

Quanto à distribuição da espécie, mesmo padrão para plantios de araucária foi observado por Rode et al. (2010) [23], que destacam também que em ambiente natural (floresta), a espécie apresenta uma distribuição muito distinta, no estudo citado apresentando uma frequência regular entre as classe. O formato da distribuição de araucária em florestas naturais é bastante variado, incluindo distribuições decrescentes [24], unimodais [14], e até bimodais, em que a curva apresenta dois pontos de maior frequência. A bimodalidade é observada por diversos autores [25, 26, 27], e decorre das características de reprodução da espécie, que apresenta ciclos endógenos de dois ou três anos [28].

Um ponto importante a ser observado nas curvas é a subestimativa ou superestimativa das classes de maior DAP das funções. Observa-se que a função Normal é a única que subestima os 
valores das últimas classes, enquanto que as outras funções modelam de maneira mais próxima ao observado essas classes de tamanho.

Ainda quanto a assimetria positiva da distribuição diamétrica de indivíduos, é importante ressaltar que apesar deste fato indicar a regeneração da floresta, segundo observado por Longhi (1980) [29], isso não garantirá a permanência da espécie, se a mesma não apresentar alta vitalidade. Apesar disto, outros estudos sugerem que, em florestas naturais, mesmo que a araucária apresente poucos indivíduos nas menores classes, a mesma apresenta condições de se manter e até aumentar o número de indivíduos, como observado por Schaaf (2006) [23] analisando a distribuição à espécie em intervalo de 20 anos.

\subsection{Relação hipsométrica}

A relação entre altura e DAP gerou um coeficiente de correlação de Pearson de 0,68 , e a tendência entre essas duas variáveis pode ser observada na Figura 2. Esse valor é considerado moderado a alto, pois, conforme Cohen (1988) [30], valores entre 0,50 e 1 podem ser interpretados como grandes. No entanto, Dancey e Reidy (2004) [31] apontam uma classificação ligeiramente diferente: $r=0,10$ até 0,30 (fraco); $r=0,40$ até 0,6 (moderado); $r=$ 0,70 até 1 (forte).

\section{Relação Altura/DAP}

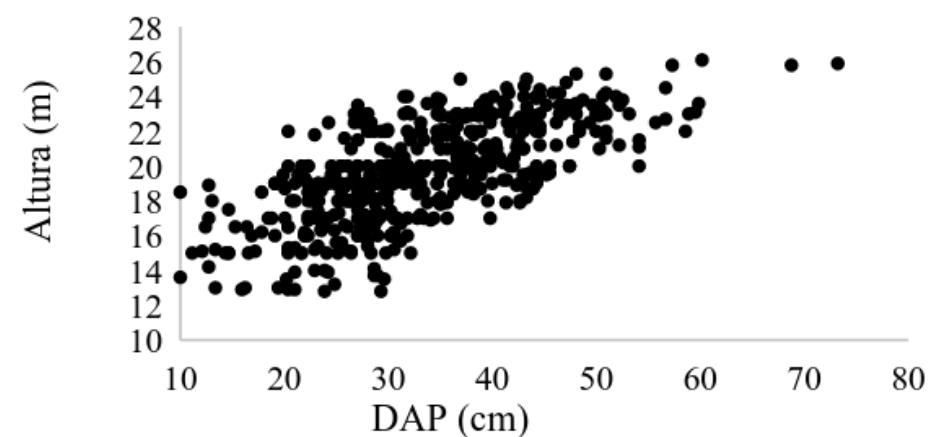

Figura 2: Relação entre as variáveis altura e DAP para Araucaria angustifolia

Os modelos foram então ajustados, e obtiveram ajustes com os coeficientes e critérios estatísticos, conforme a Tabela 4.

Tabela 4: Coeficientes e critérios estatísticos dos modelos ajustados para altura total de Araucaria angustifolia

\begin{tabular}{ccccccccc}
\hline Modelo & $\beta 0$ & $\beta 1$ & $\beta 2$ & $\beta 3$ & $\mathrm{~S}_{\mathrm{yx}}$ & $\mathrm{S}_{\mathrm{yx}}(\%)$ & IA ou R $_{\text {aj }}^{2}$ & $\mathrm{FM}$ \\
\hline 1 & 13,1273 & 0,1968 & & & 2,09 & 10,63 & 0,47 & \\
2 & 16,5166 & 0,0026 & & & 2,16 & 10,99 & 0,43 & \\
3 & 13,4408 & 0,1169 & 0,0037 & 0,0000 & 2,08 & 10,59 & 0,47 & \\
4 & $-1,1629$ & 6,0276 & & & 2,12 & 10,77 & 0,45 & \\
5 & 11,6601 & 0,2874 & $-0,0013$ & & 2,08 & 10,59 & 0,47 & \\
6 & 1,8751 & 0,3162 & & & 2,10 & 10,65 & 0,46 & 1,0063 \\
7 & 2,8061 & 0,0001 & & & 2,23 & 11,31 & 0,40 & 1,0067 \\
8 & 0,9616 & 0,5266 & 5,5895 & & 2,08 & 10,59 & 0,47 & 1,0049 \\
9 & 1,8751 & 0,1581 & & & 2,10 & 10,65 & 0,46 & 1,0063 \\
10 & 24,2469 & $-137,2617$ & & & 2,29 & 11,64 & 0,36 & \\
\hline
\end{tabular}

Em que: $\beta_{0}, \beta_{1}, \beta_{2}$ e $\beta_{3}=$ coeficientes da regressão; $S_{\mathrm{yx}}=$ Erro Padrão da Estimativa; IA = Índice de Schalaegel; $\mathrm{R}^{2}{ }_{\mathrm{aj}}=$ Coeficiente de Determinação Múltipla Ajustado; FM = Fator de Correção de Meyer.

Conforme as análises realizadas, observou-se que as equações 3, 5 e 8 obtiveram resultados iguais e as melhores performances quando observados os critérios estatísticos (IA e $\mathrm{S}_{\mathrm{yx}} \%$ ). Da 
mesma forma, a equação 1 apresentou bom ajuste, pois obteve mesmo valor de IA que as equações 8,3 e 5, porém apresentou erro ligeiramente superior.

Verifica-se que o valor do coeficiente de determinação ajustado não variou muito entre as equações testadas, estando entre os valores de 0,47 até 0,36 (equação 10). A equação 10 mostrou-se também como a pior quanto ao erro $(11,64 \%)$. Da mesma forma, o $\mathrm{S}_{\mathrm{yx}} \%$ também não variou em grande escala entre as equações, gerando a média de 10,83\%.

Em estudo realizado com Araucaria angustifolia no estado do Paraná, observou-se que o coeficiente de variação $R^{2}$ aj de 13 modelos testados ficou entre 0,17 e 0,19 [14]. Esses valores baixos significam que não houve uma boa correlação entre altura e diâmetro para os dados do estudo citado.

No presente trabalho, pode-se verificar que o $\mathrm{R}^{2}$ aj obtido foi mediano, pois apesar de ultrapassar os valores dos autores anteriormente mencionados [14], não alcançou valores altos como nos trabalhos realizados por outros autores, que observam $R^{2}$ aj de 0,89 [32] e $R^{2}$ aj de 0,83 [33], para a mesma espécie em estudo.

Além disso, segundo Batista et al. (2001) [34] o coeficiente de determinação $\mathrm{R}^{2}$ deve ser maior em florestas plantadas do que em florestas nativas. No entanto, o povoamento em questão apresentou um $\mathrm{R}^{2}$ menor do que outros estudos em plantios, realizados para Acacia mearnsii [35], para araucária [36, 37], assim como para povoamentos de Pinus sp [38], entre outros estudos.

A explicação para esses valores abaixo do esperado pode ser esclarecida mediante análise realizada em campo. As árvores do povoamento, com cerca de 60 anos, foram plantadas de forma aleatória, em diferentes sítios, caracterizando praticamente o aspecto de uma floresta nativa, e fazendo com que o povoamento crescesse de forma não homogênea, posto que apresenta árvores altas e bem desenvolvidas e outras menores, suprimidas. Além disso, a presença de espécies invasoras como do gênero Pinus e fatores como doenças, sombreamento excessivo, presença de lianas e cipós também ajudaram a impedir o crescimento em altura de alguns indivíduos.

Outro fator importante que deve ser levado em consideração, nas análises dos modelos, é que o povoamento estudado possui muitas árvores quase mortas, sem uma parte da copa ou que apresentam alguma lesão. Além disso, a baixa variação de altura em relação à amplitude diamétrica também é um fator que explica os valores de $\mathrm{R}^{2}$ aj para este trabalho.

Ainda, estudos [37] sugerem que a posição sociológica das árvores é fator importante no nível e inclinação da curva hipsométrica, e que, portanto, sugere-se que este fator deva ser considerado na construção de relações, sendo o ideal a formulação de equações que integrem essa informação ou ainda a formulação de equações para cada condição sociológica.

Para confirmar a escolha da melhor equação, recorreu-se a análises dos gráficos de dispersão das quatro equações $(1,3,5$ e 8$)$ que obtiveram os melhores valores quanto aos parâmetros estatísticos, conforme a Figura 3. Nos gráficos da Figura 3, pode-se verificar visualmente as diferenças entre as funções testadas.

Pela análise dos gráficos observa-se que todas as equações apresentam pequena tendenciosidade em subestimar os valores de altura nas menores classes de DAP. Dessa forma, como as equações foram muito semelhantes estatística e graficamente, escolheu-se a equação 5 (modelo de Trorey) como o modelo que melhor se adaptou às características da área e da população estudada, devido à simplicidade do modelo e sua ampla utilização no meio florestal. Em estudo realizado por Machado et al. (2015) [39] com Araucaria angustifolia, o modelo que se mostrou mais eficiente para a estimativa da altura total em função do DAP foi também o de Trorey. O mesmo modelo foi o mais preciso e acurado quando estudado com a espécie de Tectona grandis, considerando os critérios gerais de ajuste e a análise de resíduos, com $\mathrm{R}^{2}$ aj. de 0,89 e Syx \% de $11,37 \%$ [40]. 


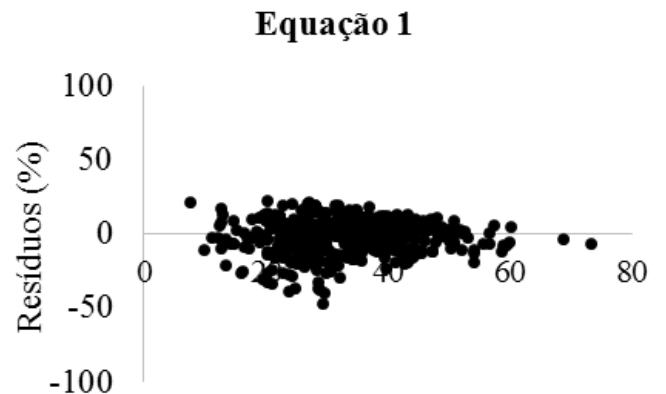

$\operatorname{DAP}(\mathrm{cm})$

Equação 5

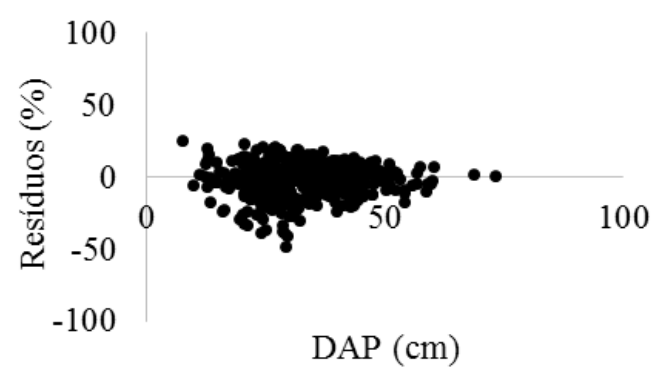

Equação 3

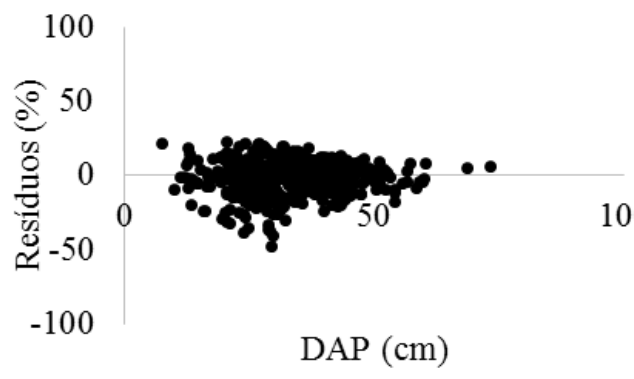

Equação 8

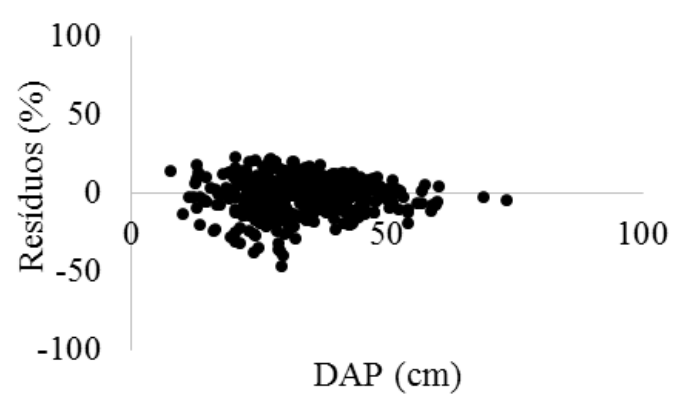

Figura 3: Gráfico dos resíduos em relação ao DAP

Os gráficos de dispersão dos resíduos possibilitam verificar que os resíduos das equações não diferiram muito entre si. Os valores dos resíduos, em relação ao DAP, variaram de $+20 \%$ a -40 $\%$. Da mesma forma, os resíduos apresentam-se bem distribuídos sob as classes de DAP. Portanto as equações podem ser utilizadas para estimar a altura total de indivíduos de Araucaria angustifolia.

Foi observado por outros autores [14], para a espécie de Araucaria angustifolia, que as distribuições gráficas dos resíduos dos modelos de Henricksen, Stoffels \& Van Soest, Chapman \& Richards (adaptada) e o modelo monomolecular (adaptado), apresentaram aproximadamente o mesmo comportamento, com os maiores valores residuais enquadrando-se entre $30 \%$ e $-30 \%$, com exceção de oito a dez árvores que apresentaram valores abaixo de $-30 \%$.

Apesar disso, deve-se levar em consideração que, segundo Batista et al. (2001) [34], a representação da curvatura da relação altura-diâmetro nos modelos pode ser considerada bastante artificial, pois grandes erros referentes a estimativa de altura podem passar despercebidos devido a extrapolações para regiões ligeiramente fora da amplitude de amostra de ajuste.

Por fim, para completar a análise, plotaram-se os pontos estimados da variável altura de cada uma das melhores equações ajustadas, juntamente com a nuvem de pontos das alturas reais das árvores, mensuradas a campo, sobre o DAP. A relação altura/diâmetro das quatro equações pode ser visualizada na Figura 4. 


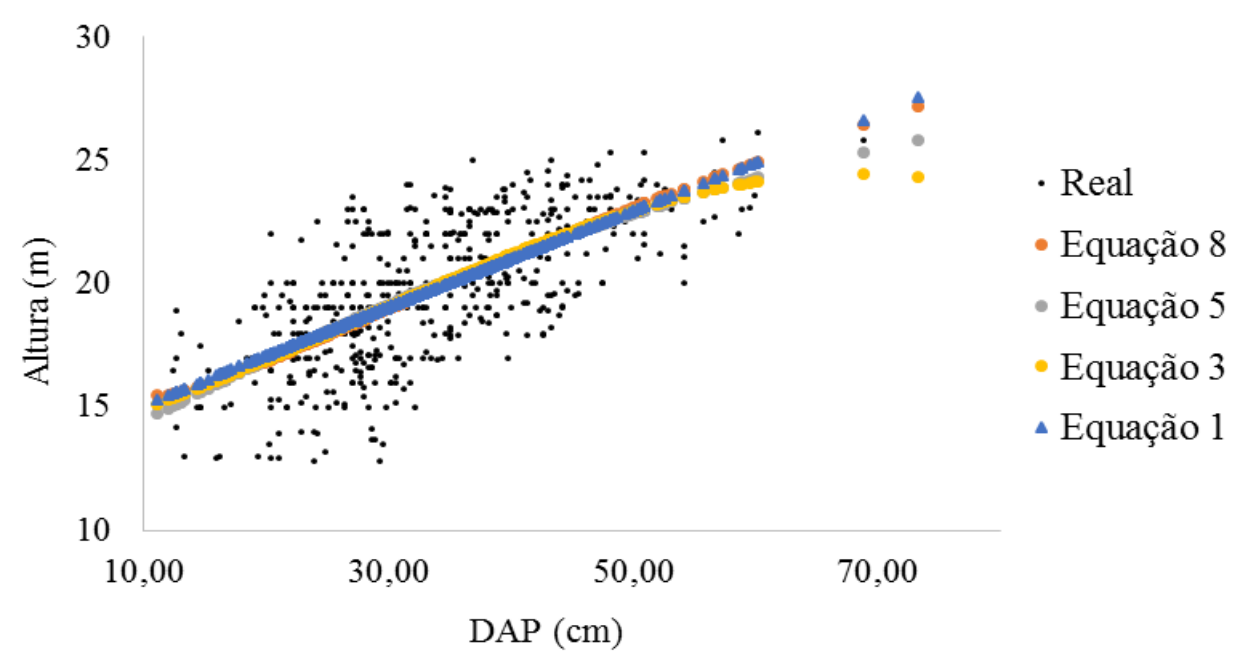

Figura 4: Relação entre os valores estimados pelas equações e os dados reais de altura.

Observa-se que o comportamento dessas linhas é bastante similar. As curvas hipsométricas são consideradas mediamente íngremes, como se espera para o estágio de desenvolvimento desse povoamento de araucária, pois, conforme Carvalho (1994) [41], as árvores dessa espécie podem atingir mais de 200 anos de idade.

A inclinação da curva fornece indícios de que este povoamento ainda está em crescimento, visto que segundo Bartoszeck et al. (2004) [18], quando a inclinação da curva é íngreme, indica que o povoamento ainda é jovem e está em desenvolvimento, e quando a inclinação é achatada, trata-se no geral de povoamentos mais velhos, onde o incremento corrente anual, principalmente em altura, está em declínio.

Outro fator considerável para explicar o comportamento dos modelos, refere-se às características de senescência do povoamento, com árvores mortas ou quase mortas, além da competição com espécies invasoras e alto grau de sombreamento, tornando o povoamento tendenciosamente como floresta natural. No geral, segundo observado por Cardoso et al. (1989) [42], povoamentos sem desbastes apresentam curvas menos íngremes em relação aos desbastados, tratando-se da espécie Pinus taeda, o que pode significar que apesar do povoamento não ser manejado, o mesmo apresenta um bom vigor.

Diversos fatores podem estar influenciando a curva hipsométrica deste povoamento, dentre eles pode-se destacar a qualidade do sítio, que é um dos fatores que influenciam a inclinação das curvas [43], sendo que, segundo Bartoszeck et al. (2004) [18], sítios mais produtivos apresentam curvas com inclinações mais acentuadas. Apesar disso, outros autores afirmam que povoamentos em sítios diferentes não apresentam diferentes inclinações, e sim diferentes níveis [44], para araucária. Já a posição sociológica apresenta-se como um fator capaz de influenciar a curva em inclinação e ponto de interceptação [37], portanto apresenta-se como um fator que pode ser responsável pela alta inclinação da curva deste povoamento.

\section{CONCLUSÃO}

As funções de densidade de probabilidade SB de Johnson e Weibull-3P foram consideradas as que apresentam melhor ajuste para os dados da distribuição diamétrica.

A relação entre altura e DAP gerou um coeficiente de correlação de Pearson de 0,68 . Já a equação $h=11,6601+874$ dap $-0,0013 d a p^{2} \operatorname{com} \mathrm{IA}=0,47 ; \mathrm{S}_{\mathrm{yx}}=2,08$ e $\mathrm{S}_{\mathrm{yx}} \%=10,59$ foi a que apresentou melhor ajuste.

A relação entre diâmetro e altura das melhores equações ajustadas apresentou inclinação mediamente íngreme, indicando que o povoamento encontra-se ainda em crescimento. 


\section{AGRADECIMENTOS}

À direção, ao corpo técnico e a todos os funcionários da FEPAGRO Florestas de Santa Maria - RS, que nos auxiliaram com materiais e orientações de campo.

À equipe do LaGeo - Laboratório de Análises Ambientais por Geoprocessamento, do DERCCR-UFSM pelo apoio nos trabalhos de campo e organização dos dados da pesquisa.

\section{REFERÊNCIAS BIBLIOGRÁFICAS}

1. Scheeren WL. Crescimento em altura de Araucaria angustifolia (Bert.) O. Ktze. em três Sítios naturais, na região de Canela - RS. Ciência Florestal. 1999; 9(2):23-40.

2. Secretaria de Estado de Meio Ambiente e Recursos Hídricos (SEMA); Instituto Ambiental do Paraná (IAP). 2008. Lista Oficial de Espécies da Flora Ameaçadas de Extinção no Paraná [Internet][acesso em dez 2014]. Disponível em: $<$ http://www.iap.pr.gov.br/arquivos/File/Atividades/POP5_LISTA_OFICIAL_ESPECIES_EXTINCA O.pdf $>$.

3. Souza AL, Jesus RM. Distribuição diamétrica de espécies arbóreas da Floresta Attântica: análise de agrupamento. Viçosa; Sociedade de Investigações Florestais; 1994. 30p.

4. Lima JPC, Leão JRA. Dinâmica de Crescimento e Distribuição Diamétrica de Fragmentos de Florestas Nativa e Plantada na Amazônia Sul Ocidental. Floresta e Ambiente. 2013; 20(1):70-79, doi: 10.4322/floram.2012.065.

5. Prodan M. Forest biometrics. Oxford: Pergamon Press; 1968. 447p.

6. Mendonça AR, Calegario N, Silva GF, Borges LAC, Carvalho SPC. Modelos hipsométricos e de crescimento em altura das árvores dominantes e codominates para Pinus caribaea var. hondurensis. Scientia Forestalis. 2011 Jun; 39(90):150-160.

7. Azevedo TL, Mello AA, Ferreira RA, Sanqueta CR, Nakajima NY. Equações hipsométricas e volumétricas para povoamento de Eucalyptus sp. localizado na FLONA do Ibura, Sergipe. Revista Brasileira de Ciências Agrárias. 2011; 6(1):105-112, doi:10.5039/agraria.v6i1a861.

8. Scolforo JRS. Biometria Florestal 2, Técnicas de regressão aplicada para estimar: volume, biomassa, relação hipsométrica e múltiplos produtos de madeira. Lavras: UFLA/FAEPE/DCF; 1997. 292 p.

9. Machado AS, Basso SF, Bevilacqua Junior VG. Teste de modelos matemáticos para o ajuste da relação hipsométrica em diferentes sítios e idades para plantações de Pinus elliottii no Estado do Paraná. $7^{\circ}$ Congresso Florestal Brasileiro; 1993; Curitiba. São Paulo: Sociedade Brasileira de Silvicultura; 1993. p. 553-556.

10. Sartori MGB. O clima de Santa Maria, RS: do regional ao urbano [dissertação]. São Paulo: Universidade de São Paulo; 1979. 165p.

11. Azevedo CP, Muroya K, Garcia LC, Lima RMB, Moura JB, Neves EJM. Relação hipsométrica para quatro espécies florestais em plantio homogêneo e em diferentes idades na Amazônia ocidental. Boletim de Pesquisa Florestal. 1999; 39:5-29.

12. Schneider PR, Finger CAG, Bernett LG, Schneider PSP, Fleig FD. Estimativa dos parâmetros da função de densidade probabilística Weibull por regressão aninhada em povoamentos desbastados de Pinus taeda L. Ciência Florestal. 2008 Jul/Set; 8(3):381-392

13. Machado AS, Bartoszeck ACPS, Figueiredo Filho A, Oliveira EB. Dinâmica da distribuição diamétrica de bracatingais na região metropolitana de Curitiba. Revista Árvore. 2006; 30(5):759-768.

14. Machado AS, Augustynczik ALD, Nascimento RGM, Fugura MA, Silva LCR, Miguel EP, Téo SJ. Distribuição diamétrica de Araucaria angustifolia (Bert.) O. Ktze. em um fragmento de floresta Ombrófila Mista. Scientia Agraria. 2009; 10(2):103-110.

15. Bartoszeck ACPS, Machado AS, Oliveira EB. A distribuição diamétrica para bracatingais em diferentes idades, sítios e densidades na região metropolitana de Curitiba. Revista Floresta. 2004 Set/Dez; 34(3):305-323, doi: 10.5380/rf.v34i3.2418.

16. Machado AS, Santos AAP, Nascimento RGM, Augustynczik ALD, Zamin NT. Modelagem da distribuição diamétrica de quatro espécies de Lauraceae em fragmento de Floresta Ombrófila Mista. Revista Ciências Exatas e Naturais. 2010 Abr/Jun; 12(1):91-105.

17. Teo SJ, Bianci JC, Peloso A, Nava PR, Marcon A, Ehlers T, Hoinacki da Costa R. Desempenho de funções de densidade probabilísticas para descrever a distribuição diamétrica de Pinus taeda, na região de caçador, SC. Floresta. 2012 Out/Dez; 42(4):741 - 754, doi: 10.5380/rf.v42i4.25976.

18. Curto RA, Mattos PP, Braz EM, Zachow R, Netto SP. Caracterização da estrutura e desenvolvimento de modelos para quantificação do potencial madeireiro em povoamento não manejado de Araucaria angustifolia. Pesquisa Florestal Brasileira. 2014 Out/Dez; 34(80):303-319, doi: 10.4336/2014.pfb.34.80.764. 
19. Figura MA. A distribuição de Weibull na descrição da estrutura diamétrica de Eucalyptus grandis: Um enfoque sobre o método dos momentos [dissertação]. Curitiba: Universidade Federal do Paraná; 2010. 98p.

20. Orellana, E, Filho AF, Netto SP, Dias AN. Modelagem da distribuição diamétrica de espécies florestais em um fragmento de floresta ombrófila mista. Revista Árvore. 2014; 38(2):297-308.

21. Amaral MK do, Netto SP, Lingnau C, Pesk VA, Martins Neto RP. Estrutura espacial de três espécies arbóreas relacionadas ao setor alimentício. Floresta. 2015; 45(1):75-84, doi: 10.5380/rf.v45i1.33679.

22. Silva VSM, Soares TS, Colpini C, Travagin DP, Hosokawa RT, Scolforo JRS. Prognose da produção de Eucalyptus camaldulensis Dehnh. Pela aplicação da função de distribuição SB de johnson. Revista Árvore. 2009; 33(5):853-863.

23. Rode R, Figueiredo Filho A, Galvão F, Machado SA. Estrutura horizontal da comunidade arbórea sob um povoamento com Araucaria angustifolia e uma Floresta Ombrófila Mista. Revista Pesquisa Florestal Brasileira. 2010 Nov/Dez; 30(64):347-361, doi: 10.4336/2010.pfb.30.64.347.

24. Schaaf LB, Fiqueiredo-Filho A, Galvão F, Sanquetta CR. Alteração na estrutura diamétrica de uma floresta ombrófila mista no período entre 1979 e 2000. Revista Árvore. 2006 Mar/Abr; 30(2):283-295, doi: 10.1590/S0100-67622006000200016.

25. Sanquetta CR. Experiências de Monitoramento no Bioma Mata Atlântica com uso de parcelas permanentes. Curitiba: RedeMap, Funpar; 2008. 338p.

26. Souza AF. Ecological interpretation of multiple population size structures in trees: The case of Araucaria angustifolia in South America. Austral Ecology. 2007; 32:524-533, doi: 10.1111/j.14429993.2007.01724.x.

27. Ebling ÂA, Péllico Netto S, Watzlawick LF, MirandaROV, Abrão SF. Ecologia e projeção diamétrica de três grupos arbóreos em remanescente de floresta Ombrófila Mista em São Francisco de Paula, RS. Floresta. 2013 Abr/Jun; 43(2):261-270, doi: 10.5380/rf.v43i2.28604.

28. Souza AF, Matos DU, Forgiarini C, Martinez J. Seed crop size variation in the dominant South American conifer Araucaria angustifolia. Acta Oecol. 2010; 36(1):126-134, doi: 10.1016/j.actao.2009.11.001.

29. Longhi SJ. A estrutura de uma floresta natural de Araucaria angustifolia (Bert.) O. Ktze, no sul do Brasil [dissertação]. Curitiba: Universidade Federal do Paraná; 1980. 198p.

30. Cohen J. Statistical power analysis for the behavioral sciences. Hillsdale: Lawrence Erlbaum Associates Publishers; 1988. 567p.

31. Dancey C, Reidy J. Estatística Sem Matemática para Psicologia: Usando SPSS para Windows. Porto Alegre: Artmed; 2004. 608p.

32. Santos WC. Análise de características dendrométricas e fatores edáficos no crescimento e produção de um povoamento de Araucaria angustifolia (Bert.) O. Ktze [dissertação]. Curitiba: Universidade Federal do Paraná; 2006. 136p.

33. Caldeira MVW, Schumacher MV, Scheeren LW, Watzlawick LF. Relação hipsométrica para Araucaria angustifolia (Bert.) O. Ktze na região oeste do estado do Paraná. Revista Acadêmica: Ciências Agrárias e Ambientais. 2003 Abr/Jun; 1(2):79-88.

34. Batista JLF, Couto HTZ, Marquesini M. Desempenho de modelos de relação hipsométrica: estudo em três tipos de floresta. Scientia Forestalis. 2001; 60:149-163.

35. Sanquetta CR, Behling A, Dalla Corte AP, Ruza MS, Simon A, São José JSB. Relação hipsométrica em inventários pré-corte em povoamentos de Acacia mearnsii De Wild. Científica. 2014; 42(1):80-90, doi: 10.15361/1984-5529.2014v42n1p80-90.

36. Sanquetta CR, Dalla Corte AP, Roglin A. Relações diâmetro-altura para espécies lenhosas em um fragmento de Floresta Ombrófila Mista no Sul do Paraná. Iheringia. 2013; 68(1):103-115.

37. Costa EA, Finger CAG, Cunha TA. Influência da posição sociológica na relação hipsométrica de Araucaria angustifolia. Revista Brasileira de Ciências Agrárias. 2014; 9(1):110-116, doi: 10.5039/agraria.v9i1a3201.

38. Araújo EJG, Pelissari AL, David HC, Miranda ROV, Netto SP, Morais VA, Scolforo JRS. Relações dendrométricas em fragmentos de povoamentos de Pinus em Minas Gerais. Pesquisa Florestal Brasileira, 2012, Out/Dez; 32(72):355-366, doi: 10.4336/2012.pfb.32.72.355.

39. Machado SA, Accioly Y, Nascimento RGM, Silva LCR da, Cardozo CC. Influência do comprimento de copa na relação hipsométrica de Araucaria angustifolia. Pesquisa florestal brasileira. 2015; 35(83): 343-351, doi: 10.4336/2015.pfb.35.83.493.

40. Sanquetta MNI, Sanquetta CR, Mognon F, Dalla Corte AP, Rodrigues AL, Maas GCB. Ajuste de equações hipsométricas para a estimação da altura total de indivíduos jovens de teca. Científica. 2015; 43(4):400-406.

41. Carvalho PER. Espécies florestais brasileiras: recomendações silviculturais, potencialidades e uso da madeira. Brasília: EMBRAPA-SPI; 1994. 640p. 
42. Cardoso DJ, Machado S do A, Rosot NC, Emerenciano DB. Avaliação da influência dos fatores idade e sítio na relação hipsométrica para Pinus taeda nas regiões central e sudoeste do estado do Paraná. Floresta. 1989; 19(1):96-115.

43. Koehler AB, Coraiola M, Netto SP. Crescimento, tendências de distribuição das variáveis biométricas e relação hipsométrica em plantios jovens de Araucaria angustifolia (Bertol.) Ktze., em Tijucas do Sul, PR. Scientia Forestalis. 2010 Mar; 38(85):53-62.

44. Figueiredo Filho A, Dias AN, Kohler SV, Verussa AA, Chiquetto AL. Evolution of the hypsometric relationship in Araucaria angustifolia plantations in the mid-south region of Paraná state. Cerne. 2010 Jul/Set; 16(3):347-357, doi: 10.1590/S0104-77602010000300010. 